\title{
Design and development of a small-scale experimental device of refrigeration system based on real faults simulation
}

\author{
Qunsheng Liu* , Lin Wang, and Huarui Cheng \\ School of Energy and Power Engineering, Henan University of Animal Husbandry and Economy, \\ 16 Bei Lin Road, Jin Shui, Zhengzhou, China \\ *liuqsh2004@163.com
}

Keywords: refrigeration system; fault simulation; real faults; filth blockage; excessively high pressure; excessively low pressure; refrigerant undercharge; refrigerant overcharge.

Abstract: Although significant progress has been made about fault detection and diagnosis (FDD) in the field of refrigeration and air conditioning, the faults that can be effectively diagnosed are still very limited. The routine maintenance and troubleshooting of refrigeration system are normally fulfilled by professional refrigeration technicians or engineers. A small-scale refrigeration system experimental device is designed and developed, as a powerful tool for teaching general theories and training practical skills for the novices of refrigeration system, This device can measure and display temperature and pressure in typical positions of a refrigeration system. In addition, it can generate real common faults for the purpose of experiments and practical training, including filth blockage, excessively high pressure, excessively low pressure, refrigerant undercharge and refrigerant overcharge. With the switching function between normal operation mode and fault operation mode, this device has been successfully applied to the college curriculums of PLC programming, refrigerating equipment maintenance and repair, etc. It is showed that the device facilitates the novices of refrigeration system to understand the changes of temperature and pressure in typical positions of a normal refrigeration system, the reasons of common faults, the methods of fault diagnosis and troubleshooting.

\section{Introduction}

Refrigeration and air conditioning plays an important role in the world economy, but also causes increasingly serious environmental concerns, such as the depletion of ozone layer and global warming. The damaging climate impact of refrigeration systems can be reduced by improving the efficiency of operation. Therefore, it is necessary to provide effective and timely maintenance and repairs to refrigeration and air conditioning systems. When a fault occurs, it is vital for a refrigeration system to find the reason of the fault rapidly, detect and determine the fault point accurately and eliminate it successfully.

On the theoretical study of fault detection and diagnosis (FDD) for refrigeration systems, lots of methods and strategies have been proposed by many investigators all over the world. Li et al. (2007) 
reported a model-based FDD in a generic way for vapor-compression air conditioners. They demonstrated that decoupling was the key to handle multiple simultaneous faults. Zhao et al. (2014) investigated a decoupling-based FDD method to deal with multiple simultaneous faults and evaluated this method in the field test environment. The field test results showed that the decoupling-based FDD method had potential to be incorporated within commercial FDD products or embedded into the control system onboard to monitor the health of the chiller's operation. Armstrong et al. (2006) developed a device they called the Non-Intrusive Load Monitor (NILM) to detect various faults in HVAC systems by observing variations in high-frequency sampled electrical data. This device can detect both mechanical and electrical related faults such as a phase loss, unbalanced voltage, refrigerant leakage, a fouled condenser coil, a dirty air filter, etc. Li et al. (2014) provided a FDD method to install variable frequency drives (VFDs) on the HVAC system, which can not only optimize the system performance, but also provide electrical parameter readings. A series of lab experiments were conducted to investigate the relationship between the electrical signals and common faults. The study also identified an electrical signature for each fault and individually separated the common faults based on three parameters: the fan power, compressor power, and the supply air temperature. Piacentino et al. (2013) proposed an improved thermoeconomic diagnosis and applied it to a reference $120 \mathrm{~kW}$ air-cooled air conditioning system. A simulator was used to evaluate thermodynamic data under normal and faulty conditions. Four faults were individually or simultaneously imposed: fouling at condenser, fouling at evaporator, refrigerant undercharge and compressor valve leakage. For setting up the diagnostic tool only a few numerical or experimental tests were required, the results testified the procedure to be sufficiently reliable both when heavy or light faults were considered. Also, the performance of the diagnostic procedure slightly improved when the effects of "system level" faults like refrigerant undercharge were preliminarily filtered. Kim et al. (2013) reported a simulation method for estimating parameters that depended on test data at rated conditions and system information. The simulation parameters decreased the errors between the actual and predicted charge. Du et al. (2014) presented a dual neural networks combined strategy to detect the faults of sensors in the supply air temperature control loop of air handling unit.

Although significant progress has been made in the field of FDD, the faults that can be effectively diagnosed are still very limited (Kocyigit et al. 2014), and many practical issues, such as cost, accuracy, reliability, robustness, and so on, are not yet well addressed. In addition, some measurements required by the FDD methods may not be commonly available on the field refrigeration and air conditioning systems. Therefore FDD remains a big challenge in the field demonstrations and commercialization of refrigeration and air conditioning systems . Because of the complication and variation of refrigeration systems, especially in the field of commercial and industrial refrigeration, the routine maintenance and troubleshooting of refrigeration system are still relied on professional refrigeration technicians or engineers, who identify the operating status of a refrigeration system, then determine the type of a fault if the status is abnormal, and eliminate the fault accordingly.

The small-scale refrigeration system experimental device designed and developed can be a powerful demonstration tool for teaching general theories and training practical skills for the novices of refrigeration system. Especially for the beginners who do not have enough background knowledge, it is very difficult for them to understand the abstract ideas of the refrigeration principles, appearances of faults, and the methods of repair, etc. Therefore, an experiment device of refrigeration, which have the function of simulating real common faults (not by electrical and other signals), and can be switched freely between the fault operation mode and the normal operation mode, can greatly facilitate the teaching of abstract theories and improve learning effects. 


\section{Principle and Procedure}

The common faults of a refrigeration system are filth blockage, excessively high pressure, excessively low pressure, refrigerant undercharge and refrigerant overcharge and so on.

Besides measuring and displaying the temperature and the pressure in the typical positions of the refrigeration system, the small-scale experiment device have the functions to fulfil five common faults which are filth blockage, excessively high pressure, excessively low pressure, refrigerant undercharge and refrigerant overcharge. These faults are the same as those appearing in actual refrigeration systems, not simulated by electrical or other signals. In addition, it can freely switch between the fault operation mode and the normal operation mode.

\subsection{Normal Operation}

The key to successful troubleshooting is the knowledge of how a refrigeration system operates and how each component functions in the system. Since a vapour-compression refrigeration system has at least four components connected by tubing, the effect of the operation of each component on the other three ones must be understood. A problem in one component may cause malfunctions in others. Hence, knowledge of refrigeration theory and the operation of the components are necessary for successful troubleshooting.

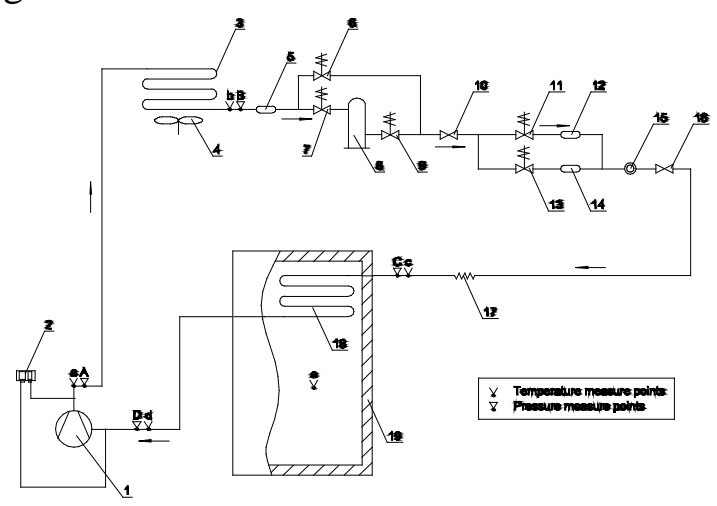

Figure 1: Schematic illustration of the experimental device (1-Compressor 2-Dual pressure control 3-Condenser 4-Fan 5, 12, 14-Filter dirers 6, 7, 9, 11, 13-Soleniod valves(normal closed) 8receiver 10,16 -Shutoff valves 15 -Sightglass 17 -Cappillary 18 -Evaporator 19 -Fridge box “ $\rightarrow$ "Flow direction of refrigerant).

As illustrated in Fig. 1, refrigerant leaves an evaporator as a low pressure, low temperature vapor and enters a compressor 1, where it is compressed into a high pressure, high temperature vapor. After that, the refrigerant enters a condenser 3, where it exchanges heat with air and turns into a high pressure, medium temperature (just above the ambient temperature, the degree of subcooling is usually about $5{ }^{\circ} \mathrm{F}$ to $9{ }^{\circ} \mathrm{F}\left[3{ }^{\circ} \mathrm{C}\right.$ to $\left.5{ }^{\circ} \mathrm{C}\right]$ ) liquid. And then the refrigerant successively flows through a filter drier 5 , a solenoid valve 7 , a receiver 8 , a solenoid valve 9 , then through a shutoff valve 10 , a solenoid valve 11, a filter direr 12 , a sightglass 15 and a shutoff valve 16 . Finally, the refrigerant flows through the capillary 17, where it is throttled into a low temperature, low pressure, gas-liquid two-phase substance, into the evaporator 18 in the box 19, vaporising and absorbing heat from the box. Then the refrigerant is recompressed and the cycle repeats.

It is not necessary to have the filter drier 5 , however, if the cleanliness of the refrigeration system is poor, particles of scale or dust or other things in pipes may be trapped by the solenoid valves when the system is operating. Thus the solenoid valve will function wrong. The purpose of setting 
shutoff valve 10 and 16 is to separate a part of the refrigeration system from the whole system conveniently when an experiment or a practical training is performed.

Shown in Table 1 are positions of measuring points of temperature and pressure for the refrigeration system of the experimental device.

Table 1 Positions of measuring points of temperature and pressure.

\begin{tabular}{|c|c|}
\hline Measuring points & Positions \\
\hline $\mathrm{a} / \mathrm{A}$ & $\begin{array}{c}\text { Outlet of the } \\
\text { compressor }\end{array}$ \\
\hline $\mathrm{b} / \mathrm{B}$ & Outlet of the condenser \\
\hline $\mathrm{c} / \mathrm{C}$ & Outlet of the capillary \\
\hline $\mathrm{d} / \mathrm{D}$ & Outlet of the evaporator \\
\hline $\mathrm{e}$ & Inside the fridge box \\
\hline
\end{tabular}

\subsection{Filth Blockage}

When the refrigeration system operates under the filth blockage mode, the solenoid valve 11 is de-energized and shuts off, meanwhile the solenoid valve 13 is energized and opens, refrigerant flows through the solenoid valve 13, the filter drier 14, the sightglass 15, etc. Except these the refrigerant flows through the same parts with that under the normal operation mode.

For the purpose of generating a real fault, a certain substance is placed at the entrance of the filter direr 14, leading to narrow flowing area. Thus, the real fault of filth blockage can be realized when the refrigerant flows through it.

\subsection{Excessively High Pressure}

Deterioration of cooling condition of a condenser can result in excessively high pressure of a refrigeration system. The most common reason is the failure of a cooling fan. The fan is deenergized when the refrigeration system is under normal operation mode. When high pressure rises, the real fault of excessively high pressure can be fulfilled. If the high pressure increases to the setting value of the pressure control, the high pressure control operates, the compressor shuts down and the pressure protection indicator light is on at the same time.

\subsection{Excessively Low Pressure}

A filth blockage fault is usually accompanied by an abnormal phenomenon of excessively low pressure. The difference between the actual low pressure and the normal low pressure depends on the degree of filth blockage. The real fault of excessively low pressure can also be realized by closing the shutoff valve manually before the capillary.

\subsection{Refrigerant Undercharge}

When the refrigeration system is under normal operation mode, the solenoid valve 6 is deenergized, the solenoid valve 7 and the solenoid valve 9 are energized. Keeping the compressor operating, let the refrigerant enter into the receiver 8 after the solenoid valve 9 is de-energized. The solenoid valve 6 is energized after a certain period of time and meanwhile the solenoid valve 7 is de-energized. From this moment the refrigerant flows through the solenoid valve 6 and the receiver is separated from the system. The real fault of refrigerant undercharge has been fulfilled because partly refrigerant which was sealed in the receiver can not circulate in the refrigeration system. 


\subsection{Refrigerant Overcharge}

When the refrigeration system is under normal operation mode, the solenoid valve 6 is deenergized, the solenoid valve 7 and the solenoid valve 9 are energized. Keeping the compressor operating, let the refrigerant stored in the receiver 8 be suctioned into the system by the compressor after the solenoid valve 7 is de-energized. The solenoid valve 6 is energized after a certain period of time and meanwhile the solenoid valve 9 is de-energized. From this moment the refrigerant flows through the solenoid valve 6 and the receiver is separated from the system. The real fault of refrigerant undercharge has been fulfilled because more refrigerant leaves from the receiver and circulates in the refrigeration system.

\section{Prototype manufacture}

As shown in Fig. 2, the box 2 adopts the insulation shell of an out-of-date household refrigerator. In order to observe the frosting process on the evaporator clearly, a copper tube-aluminum fin heat exchanger is mounted in the box as the evaporator of the experimental device instead of the original tube evaporator. The refrigerant used in the refrigeration system of the prototype is R22.

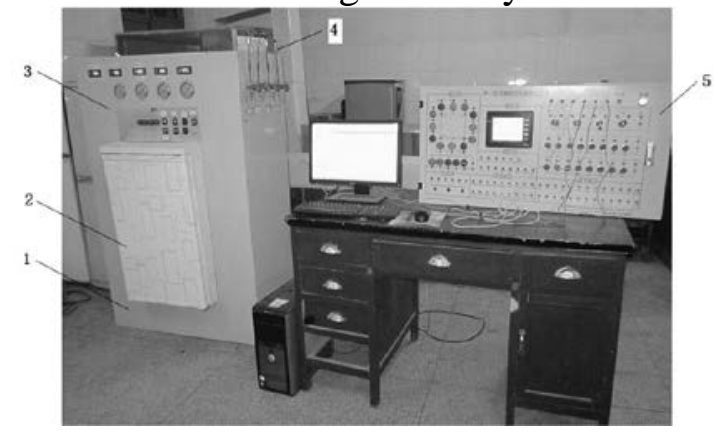

Figure 2 Experimental device (1-Frame 2-Fridge box 3-Display and control panel 4-Pressure sensor 5-Programmable logic Controller(PLC)).

As shown in the Fig. 2, four pressure sensors are set in four typical positions $A \sim D$ (shown in Fig. 1). The output signal of the pressure sensor is $4 \sim 20 \mathrm{~mA}$ DC, the experiments and practical training of automatic control and programming can be fulfilled by the Programmable Logic Controller (PLC).

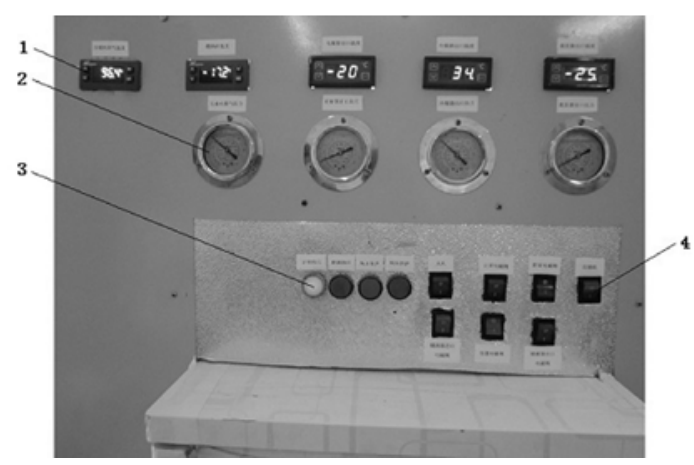

Figure 3 Display and control panel (1-Thermometers 2-Pressure gauges 3-Indicator lights 4 Control switches).

Shown in Fig. 3 is the display and control panel of the device. There are five thermometers 1, which measure and display temperature of discharge, inside of the fridge box, outlet of the capillary, outlet of the condenser and outlet of the evaporator, respectively. Four pressure gauges 2 measure and display pressure of discharge, outlet of the capillary, outlet of the condenser and outlet of the 
evaporator, respectively. Four indicator lights 3 are set to indicate the operation status of refrigeration system, representing normal operation, filth blockage, excessively high pressure and excessively low pressure, respectively. There are four control switches to control general power supply, condenser fan, solenoid valves, etc.

\section{Experimental and practical training functions and results}

\subsection{Normal Operation Mode}

It is the basic function for the experimental device operating under normal operation mode. When turn on the general power switch first, the normal operation indicator light is on, and the refrigeration system starts operating under the normal operation mode. At this time, the students can directly observe the temperature and the pressure of the four typical positions of the refrigeration system, the change of the temperature and the pressure, and frosting on the evaporator. They can also determine if the charge amount of refrigerant is enough or not simply by checking the flow of the refrigerant through the sightglass, etc.

When the ambient temperature is $79^{\circ} \mathrm{F}\left(26^{\circ} \mathrm{C}\right)$, the experiment illustrates that the device can work well. Shown in table 2 are the temperature and absolute pressure of the four typical positions of the refrigeration system of the device. The average temperature in the fridge box is $-0.8^{\circ} \mathrm{F}(-$ $\left.18.2{ }^{\circ} \mathrm{C}\right)$.

Table 2 Temperature and absolute pressure of the four typical positions of the refrigeration system.

\begin{tabular}{|c|c|c|}
\hline & $\begin{array}{c}\text { Temperature } \\
{ }^{\circ} \mathrm{F}\left({ }^{\circ} \mathrm{C}\right)\end{array}$ & $\begin{array}{c}\text { Pressure } \\
\text { psia (bar) }\end{array}$ \\
\hline Outlet of the compressor & $195(90.5)$ & $191(13.2)$ \\
\hline Outlet of the condenser & $86(30)$ & $189(13.0)$ \\
\hline Outlet of the capillary & $-13(-25)$ & $30(2.1)$ \\
\hline Outlet of the evaporator & $-8(-22)$ & $29(2.0)$ \\
\hline
\end{tabular}

\subsection{Experimental and Practical Training of the Filth Blockage Fault}

Shown in Fig.4 is a part of the refrigeration system of the device. Although the filter drier 1 shown in Fig. 4 is not necessary in principle, this component has been added for practical reason. Soldering and disconnecting copper tubes occurred many times during the trail-manufacture of the device, so the filter drier 1 is set in the refrigeration system for the consideration of safe and steady operation.

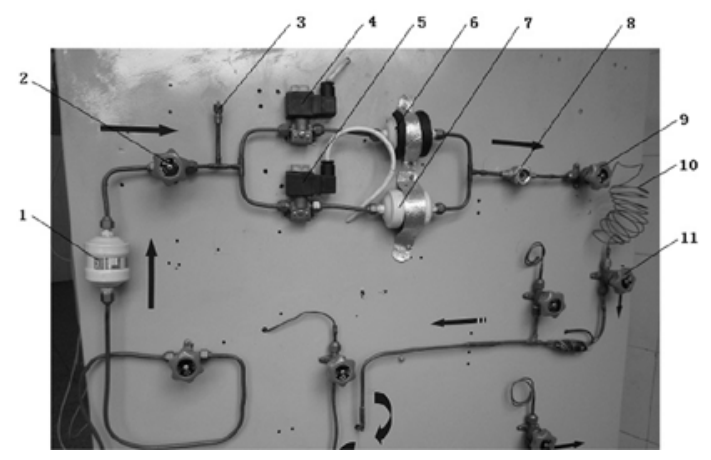

Figure 4 Partial refrigeration system of the device (1,6,7-Filter driers 2,9,11-Shutoff valves 3Service valve 4,5-Solenoid valves 8 -Sightglass 10 -Capillary). 
After the experimental device operates under the filth blockage fault about 3 minutes, the outlet of the filter drier 7 cools down evidently (but no dewing or frosting during the experiment), which is the major characteristic of the filth blockage fault. Meanwhile, low pressure drops below the normal level, and the temperature in the fridge box rises gradually, which suggests that the performance of the refrigeration system becomes worse. These phenomena subsequent to the filth blockage fault help the students to understand the reasons of the fault and to observe the phenomena of it by using the experimental device.

Once a filth blockage fault is detected, the methods and processes to eliminate the fault are as follows:

Turn off the shutoff valve 2 in Fig.4, keep the refrigeration compressor running for a certain period of time. According to the corresponding gauge when the suction pressure approximates zero, cut off the general power switch and close shutoff valve 9 quickly at the same time. After that, separate the filter direr 7 where the filth blockage fault occurs from the system, and fouling will be found at the entrance of the filter drier, which results in serious blockage. Remove the fouling and install the filter drier correctly. A nitrogen tightness experiment should be conducted through the service valve 3, and the isolation part of the system should be vacuumized carefully. Then open the closed shutoff valves and turn on the general power switch. The refrigeration system recovers.

The experimental and practical training of fault diagnosing and eliminating can be easily repeated just by placing the removed fouling at the entrance of the filter direr again.

\subsection{Experimental and Practical Training of the Excessively High Pressure Fault}

Excessively high pressure is generated by turning off the condenser fan in the experiment. The experiment shows that when the ambient temperature is $79{ }^{\circ} \mathrm{F}\left(26^{\circ} \mathrm{C}\right)$, the high pressure of the device increases within 4.5 minutes from 189 psia to 290 psia (13.0 bar to 20 bar), which is the setting value of the high pressure control. When the high pressure reaches 290 psia (20 bar), the compressor is shut down immediately. The dynamic pressure change of the device under excessively high pressure is shown in Fig.5.

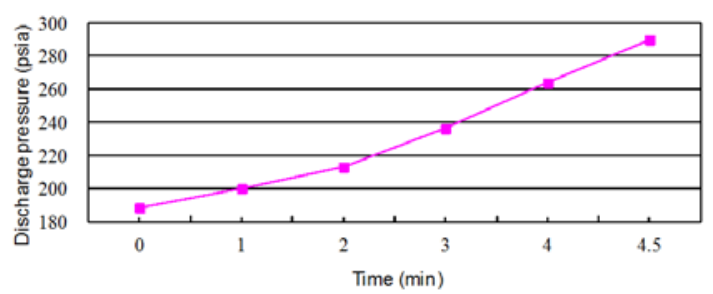

Figure 5 The high pressure varies with time during the excessively high pressure fault.

An alternative method to obtain excessively high pressure is to lay a piece of paper on the surface of air inlet of the condenser ( $\mathrm{Li}$ et al. 2009). Different value of high pressure can be obtained by controlling the cover area of the paper. This method makes it possible to achieve any level of a steady high pressure between the normal value and the setting value. Since the compressor keeps operating when the controlled high pressure changes, in addition to the high pressure, we also can observe the dynamic change of the temperature and the pressure of typical positions of the refrigeration system through the thermometers and the pressure gauges during the experiment. Therefore the theory related to the fault of excessively high pressure can be understood intensively by the students.

When the high pressure gauges show that the pressure of the outlet of the compressor or the condenser is evidently higher than the normal value, a fault of excessively high pressure occurs. The experimental device applies a dual pressure control for high pressure protection. The control 
operates when the high pressure reaches its setting value, and the high pressure protection indicator light is on to indicate that an excessively high pressure fault happens.

Now if the refrigeration system is checked, the condenser fan will be found fails. The high side of the dual pressure control used in the experimental device is an external manual reset. Therefore manually reset the control after the condenser fan operates normally to remove the fault.

\subsection{Experimental and Practical Training of the Excessively Low Pressure Fault}

The low pressure of the experiment device is approximately 29 psia (2.0 bar) when it operates normally. The setting value of the low pressure side of the dual pressure control is 3 psia ( 0.2 bar). The excessively low pressure can be generated by closing the shutoff value 9, shown in Fig. 4 . When an excessively low pressure fault occurs, the suction pressure which is considered as the low pressure normally decreases to the setting value of the control. When the control operates, cut the power supply of the compressor. The experiment shows that it takes about 50 seconds to stop the compressor after the excessively low pressure fault occurs.

When the low pressure gauges show that the pressure of the outlet of the capillary or the evaporator is evidently lower than the normal value, a fault of excessively low pressure occurs. The low pressure control operates when the low pressure reaches its setting value, and the low pressure protection indicator light is on to indicate that the excessively low pressure fault happens.

Now let's check the refrigeration system. If the excessively low pressure fault is caused by the filth blockage, it will be fixed automatically after the filth blockage fault is fixed. If the fault was caused by the closed shutoff valve before the capillary, it will be fixed after opening the valve.

\subsection{Experimental and Practical Training of the Refrigerant Undercharge Fault}

The experiment shows that it takes about 40 seconds to compress a certain mount of refrigerant into the receiver after switching the device from a normal operation mode to a refrigerant undercharge fault mode. Then, approximate 3 minutes later the visible appearances corresponding with a refrigerant undercharge fault occur on the refrigeration system of the device.

The excessively low pressure fault is generated by closing the shutoff valve 9 shown in Fig. 4 until the compressor being stopped.

The characteristics of a refrigerant undercharge fault are as follows: the cooling performance of the system deteriorates distinctly; partly frosting or even none frosting on the entire surface of the evaporator (of course, partly dewing or even none dewing for the refrigeration system whose evaporating temperature is above $32^{\circ} \mathrm{F}\left[0{ }^{\circ} \mathrm{C}\right]$ ), the low pressure and high pressure are distinctly lower than the normal levels, respectively; the suction temperature of the compressor distinctly increases, etc.

To eliminate a refrigerant undercharge fault, an appropriate amount of refrigerant should be charged into the system properly. Then, the refrigeration system recovers and the fault phenomena disappear. For the experimental device, the circulation of the refrigerant stored in the receiver implies charging an appropriate amount of refrigerant into the system.

Of course, for an actual troubleshooting of refrigerant undercharge, the reason of the fault should be checked first. The most common reason is leakage. In this case, a series of procedures should be fulfilled before charging refrigerant, such as looking for the leakage points, separating the part from the entire system, repairing the points, doing a nitrogen tightness experiment, vacuumizing the separated part of the system, etc. 


\subsection{Experimental and Practical Training of The Refrigerant Overcharge Fault}

The experiment shows that it takes about 45 seconds to suction a certain mount of refrigerant from the receiver into the refrigeration system after switching the device from a normal operation mode to an refrigerant overcharge fault mode. Then, approximate 5 minutes later the obvious appearances corresponding with a refrigerant overcharge fault occur on the refrigeration system of the device.

The characteristics of a refrigerant overcharge fault are as follows: the cooling performance of the system deteriorates distinctly; frosting from the outlet of the evaporator to the inlet of the compressor, even to the compressor head(of course, dewing instead of frosting for the refrigeration system whose evaporating temperature is above $32^{\circ} \mathrm{F}\left[0^{\circ} \mathrm{C}\right]$ ), the low pressure and high pressure are distinctly higher than the normal levels, respectively; the suction temperature of the compressor distinctly decreases, etc.

To eliminate a refrigerant overcharge fault, release some refrigerant from the system properly and recycle them by a refrigerant collector. Then, the refrigeration system recovers and the fault phenomena disappear.

\subsection{Other Experimental and Practical Training}

In addition to the experimental and practical training mentioned above, which includes normal operation, filth blockage, excessively high pressure, excessively low pressure, refrigerant undercharge and refrigerant overcharge, the experiment device can also be used for the experimental and practical training of PLC programming, vacuumizing, refrigerant charging, etc.

\section{Conclusions}

In this study, a small-scale experimental device of refrigeration system which can achieve five real faults is designed and developed. Promising experiment results and powerful teaching effects for the novices of refrigeration theory are shown as following.

- In addition to measuring and displaying the temperature and the pressure of the typical positions of the refrigeration system, the small-scale experimental device also has the functions to fulfill five real common faults, which are filth blockage, excessively high pressure, excessively low pressure, refrigerant undercharge and refrigerant overcharge. It helps the college students to learn and understand the principles and working processes of refrigeration, appearances of common faults, methods and procedures to identify and remove them. The learning interest of students, the teaching effect, the experimental and the practical training effects are improved greatly.

- The operating time from the normal operation mode to the visible appearance of any real fault stated above is all within 6 minutes. The performance of the experimental device meets the time requirement for the purpose of experiments and practical trainings.

- The device can also be used in colleges for partly experiments and practical training for the curriculums of PLC programming, refrigerating equipment maintenance and repair, etc. And it can also be used for the employee training of the companies in the field of refrigeration and air-conditioning, etc.

The experience from the development of the device shows the following aspects should be discussed and studied further.

- Since the measuring point d (shown in Fig. 1) is too close to the evaporator, the function of measuring and displaying the system superheat, which is not the superheat of the refrigerant leaving the evaporator, can't be fulfilled effectively on the device. Further improvement of the 
experiment device should be performed properly, such as putting another temperature measuring point at the inlet of the compressor.

- One of the phenomena caused by the filth blockage fault is just cooling at the outlet of the filter direr. The dewing or frosting slightly which can be observed more easily will be a better illustrative case.

- If a liquid level indicator is installed on the receiver, the condition of liquid refrigerant stored in the receiver and its leaving from the receiver into the refrigeration system will be observed more clearly and intuitively.

- For the limited budget the achievement of some real faults like refrigerant undercharge depends on controlling some switches manually, e.g. solenoids, and the timing of switch depends on experience. To make this device easier for users, the electrical control system should be improved further, e.g. a series of logical control is replaced by "One Button" of the PLC controller to perform a type of fault. However, the convenience doesn't come without cost, especially for the novices or the beginners of refrigeration theory, since the logical relationship among the series of operations would be concealed.

\section{Acknowledgements}

This research was supported by the Foundation of the Education Department Henan Province (the Key Scientific Research Project of Henan Province for Colleges and Universities, No. 15A470016).

\section{References}

[1] Armstrong, P.R., Laughman, C.R., Leeb, S.B. and Norford. L.K., 2006. Detection of rooftop cooling unit faults based on electrical measurements, HVAC\&R Research. 12 (1), pp.151-175.

[2] Du, Z.M., Fan, B., Chi, J.L. and Jin, X.Q., 2014. Sensor fault detection and its efficiency analysis in air handling unit using the combined neural networks, Energy and Building. 72, pp.157-166.

[3] Kim, W. and Braun, J.E., 2013. Performance evaluation of a virtual refrigerant charge sensor. International Journal of Refrigeration 36, pp.1130-1141.

[4] Kocyigit, N., Bulgurcu, H. and Lin, C.X., 2014. Fault diagnosis of a vapor compression refrigeration system with hermetic reciprocating compressor based on p-h diagram, International Journal of Refrigeration. 45, pp.44-54.

[5] Li, H.R. and Braun, J.E., 2007. A methodology for diagnosing multiple simultaneous faults in vapor-compression air conditioners, HVAC\&R Research. 13(2), pp.369-395.

[6] Li, H.R. and Braun, J.E., 2009. Development, Evaluation, and Demonstration of a Virtual Refrigerant Charge Sensor, HVAC\&R Research. 15(1), pp.117-136.

[7] Li, Y.H., Liu, M.S., Lau, J. and Zhang, B., 2014. Experimental study on electrical signatures of common faults for packaged DX rooftop units, Energy and Buildings. 77, pp.401-415.

[8] Piacentino, A. and Talamo, M., 2013. Innovative thermoeconomic diagnosis of multiple faults in air conditioning units: Methodological improvements and increased reliability of results, International Journal of Refrigeration. 36, pp.2343-2365.

[9] Zhao, X.Z., Yang, M. and Li, H.R., 2014. Field implementation and evaluation of a decoupling-based fault detection and diagnostic method for chillers, Energy and Buildings. 72, pp.419-430. 